\title{
Target Detection in Agriculture Field by Eigenvector Reduction Method of Cem
}

\author{
Chun-hong Liu* and Ping Li \\ College of Information and Electrical Engineering, China Agricultural University, \\ Beijing 100086, P.R. China, \\ Tel.: +86-10-82395850 \\ sophia_liu@cau.edu.cn
}

\begin{abstract}
Constrained Energy Minimization algorithm is used in hyperspectral remote sensing target detection, it only needs the spectrum of interest targets, knowledge of background is unnecessary, so it is well applied in hyperspectral remote sensing target detection. This paper analyzed the reason of better results in small target detections and worse ones in large target detections of CEM algorithm, an eigenvector reduction method to increase the ability of large target detection of CEM algorithm was proposed in this paper. Correlation matrix $\boldsymbol{R}$ was decomposed into eigenvalues and eigenvectors, then some eigenvectors corresponding to larger eigenvalues was choosed to reconstruct $\boldsymbol{R}$. In order to test the effect of the new method, experiments are conducted on HYMAP hyperspectral remote sensing image. In conclusion, by using eigenvalue reduction method, the improved CEM method not only can detect large targets, but also can well detect large/small targets simultaneously.
\end{abstract}

Keywords: Hyperspectral remote sensing, Constrained Energy Minimization algorithm, Eigenvector reduction method.

\section{Introduction}

With the development of Hyperspectral image spectrometers, hyperspectral remote sensing image can be obtained. An example of such hyperspectral sensors is 224 bands Airborne Visible Infrared Imaging Spectrometer (AVIRIS) of U.S. NASA Jet Propel Lab, another example is 210 bands HYDICE sensor [2] of Naval Research Laboratory. The resolution of an image got by high resolution spectrometers is much better than that got by multispectral sensors, people can acquire information that can not be acquired in multispectral image. This development brought hope to remote sensing target detection.

Methods based on constraint came from linearly constrained minimized variance adoptive beam-forming in digital signal processing(Chang, C.I,1999). This method also called Frost beam-forming algorithm, it constrains weight vector according to

\footnotetext{
${ }^{*}$ Corresponding author.
} 
statistic characteristic of receiving array data, when output power of beam-forming reaches minimum, beam-forming remains unit response in signal direction. Reasons of good detection in small target detection by constrained energy minimization algorithm were analyzed in this paper, then proposed an method for large targets detection based on analysis of bad reasons in large target detection. Computer simulated are carried to test the proposed algorithm.

\section{Materials and Methods}

\subsection{The Constrained Energy Minimization}

Harsanyi proposed the Constrained Energy Minimization algorithm, he considers target detection as linearly adaptive beam-forming problem(Heinz, D.C. 2000), whose characteristic is increasing pixel by pixel and separating background interfere signal from the target signal and background signal, so that target signal can be extracted in per-pixel at maximum. The step of CEM algorithm is: by designing a finite response filter, minimizing the total output energy of the linear combining process subject to a linear equality constraint applied to desired target $\boldsymbol{d}_{\circ}$ This problem can be converted to an unconstrained minimization using the method of Lagrange multipliers, using the filter to all pixel vectors to get the target detection.

Set $\boldsymbol{w}=\left[w_{1}, w_{2}, \cdots, w_{L}\right]^{T}$ as the vector of weights, where $L$ is the dimension of the data. When input is $\boldsymbol{r}_{i}=\left(r_{i 1}, r_{i 2}, \cdots, r_{i L}\right)^{T}$, then the output of filter is

$$
y_{i}=\boldsymbol{w}^{T} \boldsymbol{r}_{i}
$$

The total output energy is

$$
E=\frac{1}{N} \sum_{i=1}^{N} y_{i}^{2}=\frac{1}{N} \sum_{i=1}^{N} \mathbf{w}^{T} \mathbf{r}_{i} \mathbf{r}_{i}^{T} \mathbf{w}=\mathbf{w}^{T}\left(\frac{1}{N} \sum_{i=1}^{N} \mathbf{r}_{i} \mathbf{r}_{i}^{T}\right) \mathbf{w}=\mathbf{w}^{T} \mathbf{R} \mathbf{w}
$$

Where $N$ is all the pixel numbers in hyperspectral image data, $\boldsymbol{R}$ is sample autocorrelation matrix.

Filter $\boldsymbol{w}$ is constrained as formula (3)

$$
\boldsymbol{w}^{T} \boldsymbol{d}=1
$$

In order to get the minimum of $E$ (condition minimum), using Lagrange multiplier to compose target function $J(\boldsymbol{w})$

$$
J(\mathbf{w})=\frac{1}{2} \mathbf{w}^{T} \mathbf{R} \mathbf{w}+\beta\left(\mathbf{w}^{T} \mathbf{d}-1\right)
$$

Where $\beta$ is Lagrange multiplier, partial derivative for $\boldsymbol{w}$ and set formula (4) equal to 0 , then

$$
\frac{\partial J(\mathbf{w})}{\partial \mathbf{w}}=\frac{1}{2}\left(\mathbf{R}+\mathbf{R}^{T}\right) \mathbf{w}+\beta \mathbf{I}_{L} \mathbf{d}=\mathbf{0}
$$


Where $\boldsymbol{I}_{\mathrm{L}}$ is $L \times L$ unit matrix, because $\boldsymbol{R}$ is a symmetrical matrix and non-singular, then

$$
\boldsymbol{w}=-\beta \boldsymbol{R}^{-1} \boldsymbol{d}
$$

Put formula (6) into formula (3), $\beta$ can be decided

$$
\beta=-\left(\boldsymbol{d}^{T} \boldsymbol{R}^{-1} \boldsymbol{d}\right)^{-1}
$$

Put formula (7) into formula (6), the filter coefficient $\mathbf{w}_{C E M}$ is

$$
\mathbf{w}_{C E M}=\frac{\mathbf{R}^{-1} \mathbf{d}}{\mathbf{d}^{T} \mathbf{R}^{-1} \mathbf{d}}
$$

By using filter coefficient in formula (8), a CEM detector can detect desired target $\boldsymbol{d}$ by using $C E M(\boldsymbol{r})=\boldsymbol{w}_{C E M}^{T} \boldsymbol{r}$, at the same time, minimum output energy caused by interfere background and unknown signal.

\subsection{Analysis of Good Detection for Small Targets by CEM}

By applying CEM algorithm into hyperspectral remote sensing target detection, CEM algorithm can detect small target better, the reason is analyzed as following.

Essentially, CEM can be seem as the inverse of Principal Component Analysis. By using PCA, main direction of original data distribution can be got, that is several eigenvectors corresponding to large eigenvalues can be decided(Geng X.R.2005). That is, PCA compresses most meaningful information from original image feature space to space that several non-correlation principals composed. Obviously, targets with low probability(small targets) in image will not included in these principals, on contrary, they often appear at feature direction corresponding to small eigenvalues of correlation matrix (Joseph C. H. 1993).

CEM finds targets by calculating $C E M(\boldsymbol{r})=\boldsymbol{w}_{C E M}^{T} \boldsymbol{r}$, if there are small targets whose corresponding energy are small, and they are corresponding to small eigenvalues of autocorrelation matrix $\boldsymbol{R}$. while smaller the eigenvalue, $C E M(\boldsymbol{r})=\boldsymbol{w}_{C E M}^{T} \boldsymbol{r}$ the bigger, that is the reason why CEM can detect small targets. The above discuss can be deduced as following.

Decomposing $\boldsymbol{R}$ into eigenvalues and eigenvectors

$$
\boldsymbol{R}=\boldsymbol{V} \boldsymbol{\Lambda} V^{T}
$$

where $\boldsymbol{\Lambda}=\operatorname{diag}\left(\lambda_{1}, \cdots, \lambda_{i}, \cdots, \lambda_{L}\right)$ is $L \times L$ diagonal matrix which contains eigenvalues of $\boldsymbol{R} \cdot \boldsymbol{V}=\left(\boldsymbol{v}_{1}, \cdots, v_{2}, \cdots, v_{L}\right)$ is an $L \times L$ normalized diagonal matrix whose columns are eigenvectors of $\boldsymbol{R}$. Then

$$
\boldsymbol{R}^{-1}=\boldsymbol{V} \boldsymbol{\Lambda}^{-1} \boldsymbol{V}^{T}
$$

$\lambda_{i}$ ever larger, $\boldsymbol{R}^{-1}$ ever smaller, then the smaller of the value, the ability of CEM detecting small targets get worse, while the ability of detecting large targets increased. 
$\lambda_{i}$ ever small, $\boldsymbol{R}^{-1}$ ever larger, then the bigger of the value, the ability of CEM detecting small targets increased, while the ability of detecting large targets get worse.

In conclusion, if all eigenvalues of $\boldsymbol{R}$ are adopted, that is, eigenvector corresponding to small eigen-values are included into detector, then CEM will get good detection results.

\subsection{Revision of CEM Tetection Large Targets}

By observing the format of CEM, the performance of the CEM technique for target detection is strongly dependent on the structure of the sample correlation matrix $\boldsymbol{R}$. The ability to calculate the inverse of $\boldsymbol{R}$ accurately is a critically important consideration when applying the CEM operator.

The eigen-decomposition of $\boldsymbol{R}$ is given by

$$
\boldsymbol{R}=\boldsymbol{V} \boldsymbol{\Lambda} V^{T}
$$

where $\Lambda=\operatorname{diag}\left(\lambda_{1}, \cdots, \lambda_{i}, \cdots, \lambda_{L}\right)$ is an $L \times L$ diagonal matrix containing the eigenvalues of $\boldsymbol{R}$, and $\boldsymbol{V}=\left(\boldsymbol{v}_{1}, \cdots, \boldsymbol{v}_{2}, \cdots, \boldsymbol{v}_{L}\right)$ is an $L \times L$ unitary matrix whose columns are the eigenvectors of $\boldsymbol{R}$. Eigenvalue $\lambda$ represents the size of target projection to feature space, eigenvector is the direction of target project on feature space (Shen F.L. 2001). According to the above analysis, in order to detect large target, a good estimate of the correlation matrix can be obtained by only considering the contribution of the first significant eigen vectors where $p$ is an estimate of the intrinsic dimensionality of the hyperspectral data. Considering the contribution of only the first $p$ eigenvectors, the correlation matrix estimate is given by

$$
\hat{\boldsymbol{R}}=\tilde{\boldsymbol{V}} \tilde{\boldsymbol{\Lambda}} \tilde{\boldsymbol{V}}^{T}
$$

Where $\tilde{\boldsymbol{V}}=\left(\boldsymbol{v}_{1}, \cdots, v_{i}, \cdots, v_{p}\right)$ is the $L \times p$ matrix whose columns are the significant eigenvectors and $\tilde{\Lambda}=\operatorname{diag}\left(\lambda_{1}, \cdots, \lambda_{i}, \cdots, \lambda_{p}\right)$ is a $p \times p$ diagonal matrix.. An estimation of $\boldsymbol{R}^{-1}$ is given by

$$
\hat{\boldsymbol{R}}^{-1}=\tilde{\boldsymbol{V}} \tilde{\boldsymbol{\Lambda}}^{-1} \tilde{\boldsymbol{V}}^{T}
$$

where $\tilde{\boldsymbol{\Lambda}}^{-1}=\operatorname{diag}\left(\lambda_{1}^{-1}, \cdots, \lambda_{i}^{-1}, \cdots, \lambda_{p}^{-1}\right)$. Replacement of $\boldsymbol{R}^{-1}$ in CEM target detection operator with $\hat{\boldsymbol{R}}^{-1}$ will provide the desired results for large targets.

After eigen decomposition, the decomposed matrix is not only helpful to understand geometry constructions of different classes, but also helpful to understand classification of high dimension space.

Because big eigenvalue represents big targets, autocorrelation $\hat{\boldsymbol{R}}$ are composed by several big eigenvalues and eigenvectors, large targets can be detected better. 


\subsection{Experiment Simulation}

\subsubsection{Experiment Image}

The experiment image is 126 bands HYMAP hyperspectral remote sensing image. The data showed part of Indiana remote sensing test region in U.S. in September 1999, it contains agriculture and forest, etc. the characteristic of HYMAP data showed in table1. , a part of data was used in this experiment. Pseudo image of band 32、16、 8 used as RGB channel, 4 interest targets was selected, P1 and P2 are small targets, $\mathrm{P} 3$ and $\mathrm{P} 4$ are big targets.

Table 1. Characteristic of HYMAP data used in the experiment

\begin{tabular}{ll}
\hline Characteristic & Parameter \\
\hline Image size & $151 \times 266$ pixel \\
Fly altitude & 1500 meter \\
Resolution & $3 \times 3$ square \\
Pixel depth & 16 bits \\
Band number & 126 \\
Spectral domain & $0.45-2.5 \mu \mathrm{m}, 3-5 \mu \mathrm{m} \quad 8-12 \mu \mathrm{m}$ \\
Spectral resolution & VIS-SWIR:10-20nm, TIR: $100-200 \mathrm{~nm}$ \\
\hline
\end{tabular}

\subsubsection{Experiment Results}

Correlation matrix $\boldsymbol{R}$ of all the pixels in HYMAP image was decomposed in this experiment. According to big to small, eigenvectors corresponding to all eigenvalues: first 100 , first 50 , first 10 , first 5 respectively are used to construct approximate $\hat{\boldsymbol{R}}$, the detection results showed in Fig.2 Fig.6. From the detection results, we can see, when all eigenvectors are used, CEM detector can get good detecting results for small targets, while worse detection results for large target; along with the reduction of eigenvectors, that is, eigenvectors reduction proposed in this paper, CEM target detector get worse results for small targets, while better results for large targets.

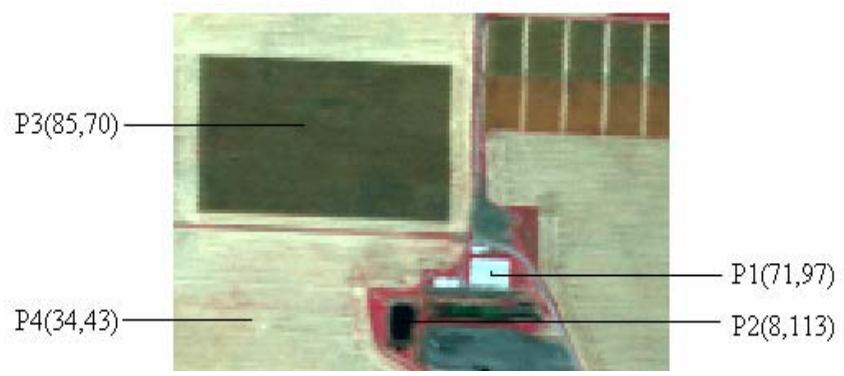

Fig. 1. Pseudo image of band 32, 16, 8 and interest targets selected 


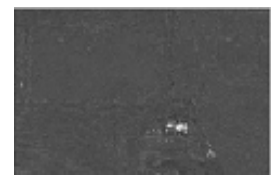

(a) target1

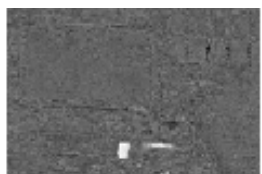

(b) target 2

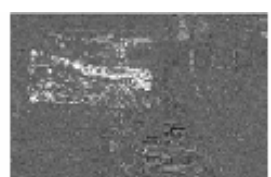

(c) target 3

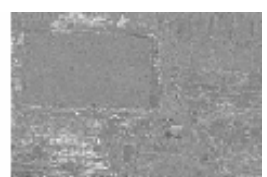

(d) target 4

Fig. 2. Detection results by all the eigenvectors of $\boldsymbol{R}$

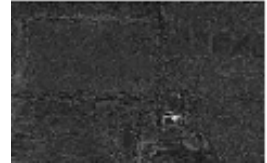

(a) target1

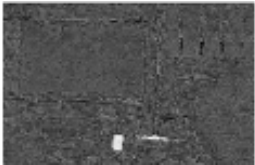

(b) target 2

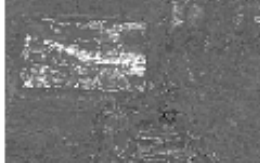

(c) target 3

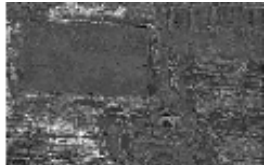

(d) target 4

Fig. 3. Detection results by first 100 eigenvectors of $\boldsymbol{R}$

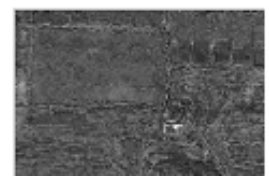

(a) target1

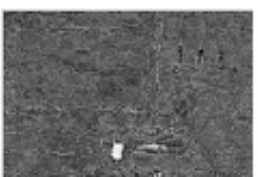

(b) target 2

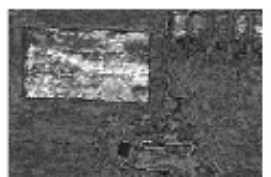

(c) target 3

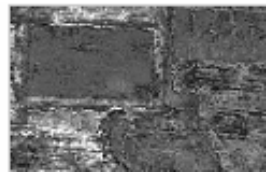

(d) target 4

Fig. 4. Detection results by first 50 eigenvectors of $\boldsymbol{R}$

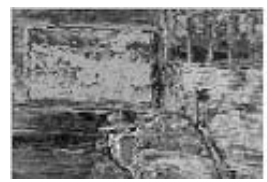

(a) target1

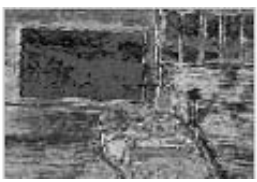

(b) target 2

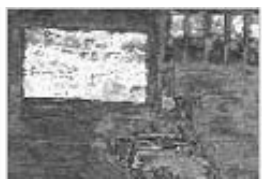

(c) target 3

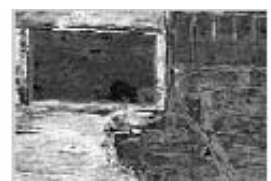

(d) target 4

Fig. 5. Detection results by first 10 eigenvectors of $\boldsymbol{R}$

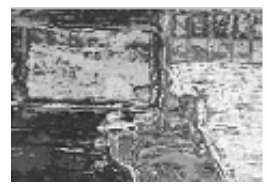

(a) target 1

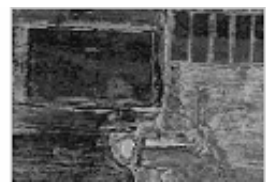

(b) target 2

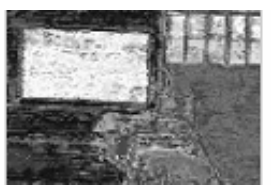

(c) target 3

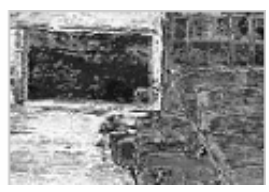

(d) target 4

Fig. 6. Detection results by first 5 eigenvectors of $\boldsymbol{R}$ 


\section{Conclusion}

An eigenvector reduction method was proposed in this paper, and HYMAP hyperspectral remote sensing data was used to test this new method. Autocorrelation matrix $\boldsymbol{R}$ was decomposed into eigenvalues and eigenvectors, then eigenvalues were selected from big to small, and corresponding eigenvectors were composed into new autocorrelation matrix. By computer simulations, three conclusions were got:

(1) When all eigenvalues and eigenvectors are used, CEM remains its primary characteristic, that is detecting small targets well;

(2) When half eigenvectors corresponding to larger eigenvalues were used, both small and large targets can be detected well;

(3) When seldom eigenvectors corresponding to larger eigenvalues were used, large targets can be detected well.

In conclusion, by using eigenvalue reduction method, the improved CEM method not only can detect large targets, but also can well detect large/small targets simultaneously. When application, we can select all or part of eigenvectors flexibly in order to get good detection results for large or small targets.

\section{References}

Chang, C.I., Hsuan, R.: Linearly Constrained Minimun Variance Beamforming Approach to Target Detection and Classification for Hyperspectral Imagery. In: Proceedings of Geoscience and Remote Sensing Symposium, IGARSS 1999, vol. 2, pp. 1241-1243. IEEE, Los Alamitos (1999)

Chang, C.I., Heinz, D.C.: Constrained subpixel target detection for hyperspectral imagery.

In: Proceedings of SPIE - The International Society for Optical Engineering, vol. 4048, pp. 35-45 (2000)

Geng, X.R.: Research of target detection and classification of hyperspectral remote sensing. Ph.D Dissertation, pp. 81-87 (2005)

Joseph, C.H.: Detection and Classification of Subpixel Spectral Signatures in Hyperspectral Image Sequenecs. Ph.D Dissertation, Maryland University, USA, pp. 84-89 (1993)

Shen, F.L., Ye, Z.F., Qian, Y.M.: Signal statistic analysis and processing, pp. 561-562. University of Science and Technology of China Press (2001) 